heterogeneity, because it can be easily matched with HER2 IHC slide under light microscope. We agree to Arena et al. that detailed report of HER2 IHC is the simplest way to report HER2 regional heterogeneity. As shown in our study, heterogeneous HER2 expression was well matched with HER2 regional or genetic heterogeneity.

The main issue pointed out by Chang is the prognostic significance of HER2 intratumoral heterogeneity. He argued that the conflicting prognostic effect of HER2 intratumoral heterogeneity is related to patient treatment. We agree that different treatment may account for different outcome, as shown in the study by Bartlett et al. ${ }^{5}$ For survival analysis, they used TEAM (Tamoxifen vs Exemestane adjuvant Multicentre) pathology study composed of estrogen receptor-positive early breast cancers, treated with adjuvant endocrine therapy (exemestane versus tamoxifen), but not with chemotherapy. ${ }^{6}$ However, tumors with heterogeneous HER2 amplification cannot be equated with those with borderline/low HER2 amplification. Although HER2 genetic and regional heterogeneity was more common in tumors with equivocal/low-grade amplification, they were found in 23 and $38 \%$ of tumors with equivocal/lowgrade amplification in our study. Thus, comparison of our study with the HERA trial ${ }^{7}$ is not reasonable. We admit that our study has a limitation for survival analysis due to small number of heterogeneous cases. Further large-scaled, well-designed studies will be needed to find clinically relevant definition of HER2 intratumoral heterogeneity and to establish its prognostic significance and predictive value for HER2 targeted therapy.

\section{Disclosure/conflict of interest}

The authors declare no conflict of interest.
Hee Jin Lee ${ }^{1}$ and So Yeon Park ${ }^{1,2}$

${ }^{1}$ Department of Pathology, Seoul National University Bundang Hospital, Seongnam, Gyeonggi, Korea; ${ }^{2}$ Department of Pathology, Seoul National University College of Medicine, Seoul, Korea E-mail: sypmd@snu.ac.kr

\section{References}

1 Seol H, Lee HJ, Choi Y, et al. Intratumoral heterogeneity of HER2 gene amplification in breast cancer: its clinicopathological significance. Mod Pathol 2012; 25:938-948.

2 Vance GH, Barry TS, Bloom KJ, et al. Genetic heterogeneity in HER2 testing in breast cancer: panel summary and guidelines. Arch Pathol Lab Med 2009;133: 611-612.

3 Layfield LJ, Schmidt RL. HER2/neu gene amplification heterogeneity: the significance of cells with a 3: 1 HER2/ CEP17 ratio. Appl Immunohistochem Mol Morphol 2012;20:543-549.

4 Allison KH, Dintzis SM, Schmidt RA. Frequency of HER2 heterogeneity by fluorescence in situ hybridization according to CAP expert panel recommendations: time for a new look at how to report heterogeneity. Am J Clin Pathol 2011;136:864-871.

5 Bartlett AI, Starcyznski J, Robson T, et al. Heterogeneous HER2 gene amplification: impact on patient outcome and a clinically relevant definition. Am J Clin Pathol 2011;136:266-274.

6 Bartlett JM, Brookes CL, Robson T, et al. Estrogen receptor and progesterone receptor as predictive biomarkers of response to endocrine therapy: a prospectively powered pathology study in the Tamoxifen and Exemestane Adjuvant Multinational trial. J Clin Oncol 2011;29:1531-1538.

7 Dowsett M, Procter M, McCaskill-Stevens W, et al. Disease-free survival according to degree of HER2 amplification for patients treated with adjuvant chemotherapy with or without 1 year of trastuzumab: the HERA Trial. J Clin Oncol 2009;27:2962-2969.

\title{
DDIT3, STT3A (ITM1), ARG2 and FAM129A (Niban, C1orf24) in diagnosing thyroid carcinoma: variables that may affect the performance of this antibody-based test and promise
}

\author{
Modern Pathology (2013) 26, 611-613; doi:10.1038/modpathol.2012.212
}

To the editor: We have read with special interest, the paper by Sigstad et $a l^{1}$ published in a recent issue of Modern Pathology (25: 537-547, 2012). The authors sought to confirm whether ITM1 (alias STT3A), C1orf24 (aliases FAM129A and Niban), DDIT3 (alias GADD153) and ARG2 could discriminate follicular thyroid carcinoma (FTC) from follicular thyroid adenoma (FTA). In their study, they were not able to use these markers to discriminate between FTC and FTA.
In our previous work we found that these markers would discriminate between FTA and FTC with high sensitivity and specificity. Custom antibodies for ITM1 and C1orf24 and commercially available ARG2 and DDIT3 antibodies were tested on thyroid lesions commonly diagnosed as indeterminate by fine-needle aspiration biopsy. We showed that immunohistochemistry (IHC) was more sensitive than quantitative PCR in detecting thyroid carcinomas, and that the combination of four markers was 
more effective than a single marker. ${ }^{2}$ Although the antibody-based test had high sensitivity (1.0), the specificity was lower (0.85). The false-positive cases were Hürthle cell adenomas (HCA), which were positive for all four carcinoma markers. ${ }^{3}$ To locate new HCA markers, in part to identify these false positives, global gene expression profiling of a HCA and a Hürthle cell carcinoma was performed. PVALB was identified as a new HCA marker, as it was negative in a wide range of thyroid carcinomas and was positive in HCA. ${ }^{4}$ This validation analysis was consistent with our previous findings and also demonstrated that C1orf24 is the best predictor among the four carcinoma markers, followed by ITM1. ${ }^{2}$

From the study by Sigstad et al ${ }^{1}$ it might be concluded that there is no clinical utility for these markers to distinguish between FTA and FTC. We take this opportunity to call attention to some variables that can influence test results and, therefore, why these markers might be useful for an important clinical problem and should be further tested.

One important variable that may account for the conflicting results is that different antibodies for some of the genes were used. We used customproduced antibodies for ITM1 and C1orf24. Moreover, Sigstad and coworkers' antibodies were generated using peptide conjugated to diphetheria toxoid, whereas in our study the antibodies were conjugated to keyhole limpet hemocyanin by the lysine at the C-terminus. The selection of the carrier protein and coupling strategy can greatly influence the antibodies titer and specificity.

To check for specificity, it is important to use appropriate controls, as well as the use of independent methods. One appropriate first validation step is overexpressing transfected cell lines compared with control cells that lack the gene. To demonstrate specific antibody binding, cells transfected with the proteins of interest were our positive controls, whereas parental cells were negative controls. The predicted protein size was clearly observed in originally C1orf24-free NIH3T3 cells that were transfected with an expression vector containing the cDNA of C1orf24. The protein was not detected in normal thyroid tissues and parental cells. Interestingly, a band of nearly $70 \mathrm{kDa}$ was detected in an FTC sample. Importantly, Adachi et $a l^{5}$ also produced antibodies using C-terminal peptides comparable to the one used by our group. The protein was observed in NIH3T3 cells expressing C1orf24 and human renal carcinoma cell lines, whereas were not observed in normal kidney. The authors suggested that the small size of C1orf24 protein may correspond to C1orf24 that have undergone processing under stress conditions such as oxidative stress and hypertonic stress. ${ }^{5}$

Sigstad et $a l^{1}$ showed that the polyclonal antibody against C1orf24 successfully identify the expression of a $130 \mathrm{kDa}$ protein in a follicular carcinoma cell line (FTC 133) and a FTC sample. However, the protein was also detected in 3/5 FTAs and in two normal thyroids. ${ }^{1}$ We recently evaluated its expression in a paired primary thyroid follicular carcinoma (FTC 133) and lymph node metastatic cell lines (FTC 236) and a FTC cell line (WRO). In FTC 236 and WRO both the 130 and $70 \mathrm{kDa}$ bands were observed, whereas in FTC 133 cell line only a $70 \mathrm{kDa}$ band was observed. These findings, compared with the Sigstad et al, ${ }^{1}$ suggest that different epitopes may have been recognized by C1orf24 antibodies (data not shown).

Regarding the ITM1 antibody, the predicted size is $80 \mathrm{kDa}$. Sigstad et al ${ }^{1}$ detected a $60 \mathrm{kDa}$ band in both cell line and thyroid tissues. These findings suggest the possibility that the ITM1 antibody employed in their study and the one used in our study may have detected different epitopes on the same protein or may have recognized epitopes from different proteins.

Importantly, an antibody may work in a western blot assay but fail in IHC. When the ultimate goal is to use the antibody for IHC, the user must demonstrate that the antibody is also able to specifically identify its target on paraffin-embedded sections.

Although a negative control including no primary antibody is valuable for the specificity of the secondary antiserum, a negative control would be a tissue that is known to not express the protein of interest. Not only the expression of these markers was absent in normal thyroid SAGE library but also these proteins were negative in all IHC validation steps. $^{2}$ That is an important issue, as the presence of cross-reactive substances in normal thyroid would refute the diagnostic value of these markers because fine-needle aspiration specimens will contain normal thyroid cells. Hence, normal thyroid should be included as negative control in all validation steps. This concern should be better addressed in the Sigstad et $a l^{1}$ study as nearly all samples were positive for all antibodies. In their study, it is not clear which sample and/or tissue was used for the titration of the antibodies and used as positive and negative control.

There were substantial differences between our studies and the Sigstad study that may account for different results. In addition to aforementioned factors, variables such as the time before fixation, the length of fixation time, differences in fixatives and tissue processing can all affect tissue antigenicity and the status of the epitope of interest. Furthermore, if the antibody has not been affinity purified, it may produce a high background on tissues. For instance, in our study we used affinitypurified antibodies, reduced fixation time before embedding in paraffin, reduced incubation period of primary antibody and the peroxidase-quenching step was performed using 100-fold higher concentration. These variables may also explain their inconsistent results, as when the authors used tissue microarray samples purchased from US Biomax, higher specificity and sensitivity was obtained. 
Sigstad et $a l^{1}$ conclusion, that this panel of markers is not valid to distinguish FTA and FTC, was also based on Matsumoto et al ${ }^{6}$ data. However, in their manuscript, C1orf24 was expressed in nearly all PTC and 50\% of FTC, whereas was not expressed in normal thyroid. Notably, we have also emphasized that none of these markers should be used alone.

We also share with other groups a strong belief that C1orf24 has a role in the pathogenesis of other tumor subtypes. Ito et $a l^{7}$ found that C1orf24 was positive in $97 \%$ of head and neck squamous cell carcinoma and in $66 \%$ of dysplastic lesion, whereas was not observed in normal mucosal samples. It was also identified as a new candidate marker for renal carcinogenesis, as it was positive in sporadic human renal carcinoma cell lines and not observed in normal kidney. ${ }^{5}$ Finally, functional analyses have supported that C1orf24 have a potential role in protecting cells from genotoxic stress-induced apoptosis. ${ }^{8}$ In addition, they demonstrated that C1orf24 depletion increased cell apoptosis. The authors suggest that AKT contributes to resistance of cancer cells through C1orf24 phosphorylationpromoted P53 degradation. ${ }^{8}$

It is clear that there is a need to perform more studies using this panel of markers. A practical challenge to demonstrate the clinical application of our proposed antibody-based test is that we had used custom-produced antibodies. Currently, however, there are few companies that generated antibodies against ITM1, C1orf24 and PVALB. Unquestionably, a study designed to compare antibodies of various sources, including all commercially available and custom produced, should be performed to determine antibodies specificity and reproducibility and, therefore, whether they are useful markers that can be applied into clinical practice. However, one should have in mind that one batch of an antibody may work, whereas the next, with a different lot, may not. As antibodybased test will be widely used on clinical decisions, standardization and development of antibody guidelines are critical. For the meantime, if of our responsibility to have our eyes open to all potential problems and assure that the results are replicable and likely to be correct.

\section{Disclosure/conflict of interest}

The authors declare no conflict of interest.

Gianna M Carvalheira, Bruno HN Nozima, Gregory J Riggins and Janete M Cerutti Laboratório a Base Genética dos Turmores da Tiroide, Pedro de Toledo 669, 11 andar, Department of Morphology and Genetics, Federal University of São Paulo, 04039-032, São Paulo, SP, Brazil E-mail: j.cerutti@unifesp.br

\section{References}

1 Sigstad E, Paus E, Bjoro T, et al. The new molecular markers DDIT3, STT3A, ARG2 and FAM129A are not useful in diagnosing thyroid follicular tumors. Mod Pathol 2012;25:537-547.

2 Cerutti JM, Delcelo R, Amadei MJ, et al. A preoperative diagnostic test that distinguishes benign from malignant thyroid carcinoma based on gene expression. J Clin Invest 2004;113:1234-1242.

3 Cerutti JM, Latini FR, Nakabashi C, et al. Diagnosis of suspicious thyroid nodules using four protein biomarkers. Clin Cancer Res 2006;12:3311-3318.

4 Cerutti JM, Oler G, Delcelo R, et al. PVALB, a new Hurthle adenoma diagnostic marker identified through gene expression. J Clin Endocrinol Metab 2011;96:E151-E160.

5 Adachi $\mathrm{H}$, Majima S, Kon S, et al. Niban gene is commonly expressed in the renal tumors: a new candidate marker for renal carcinogenesis. Oncogene 2004;23:3495-3500.

6 Matsumoto F, Fujii H, Abe M, et al. A novel tumor marker Niban, is expressed in subsets of thyroid tumors and Hashimoto's thyroiditis. Hum Pathol 2006;37:1592-1600.

7 Ito S, Fujii H, Matsumoto T, et al. Frequent expression of Niban in head and neck squamous cell carcinoma and squamous dysplasia. Head Neck 2010;32:96-103.

8 Ji H, Ding Z, Hawke D, et al. AKT-dependent phosphorylation of Niban regulates nucleophosmin- and MDM2-mediated p53 stability and cell apoptosis. EMBO Rep 2012;13:554-560.

\title{
Reply to 'The new molecular markers DDIT3, STT3A, ARG2 and FAM129A are not useful in diagnosing thyroid follicular tumors'
}

\author{
Modern Pathology (2013) 26, 613-615; doi:10.1038/modpathol.2013.39
}

To the Editor: In reply to Carvalheira et al's ${ }^{1}$ letter regarding our article, ${ }^{2}$ we would like to answer as follows:

First, considering the urgent need for markers to distinguish between follicular adenoma (FTA) and carcinoma (FTC) during FNA cytology, we find it remarkable that no clinical studies have been published validating the four classifiers originally described by Cerutti et al. ${ }^{3}$ Indeed, we are unaware of any diagnostic laboratory using these markers 